\title{
Prevalence of Poly-pharmacy in the Elderly: Implications of Age, Gender, Co-morbidities and Drug Interactions
}

\author{
Mubarak N. Al Ameri ${ }^{1,2 *}$, Emad Makramalla ${ }^{1 \#}$, Umniya Albur $^{1 \#}$, Anil Kumar ${ }^{1 \#}$ and \\ Padma Rao $^{3 \#}$ \\ ${ }^{1}$ Abu Dhabi Hospital, Global Medical Solutions, United Arab Emirates \\ ${ }^{2}$ William Harvey Research Institute, London, UK \\ ${ }^{3}$ Clinical Pharmacy and Pharmacology Department, RAKCOPS, RAKMHSU, RasAlkhaima, UAE \\ "Authors contributed equal for this research
}

Received: August 02, 2014, Accepted: September 01, 2014, Published: September 09, 2014

*Corresponding author: Mubarak N. Al Ameri, Abu Dhabi Hospital, Global Medical Solutions, UAE, E-mail: m.n.alameri@gmail.com

\begin{abstract}
Poly-pharmacy is defined as the concurrent use of five or more medications and is critical in individuals with multiple chronic medical conditions. There are many factors affecting the incidence of poly-pharmacy. This study will assess the relationship between poly-pharmacy and factors such as age, gender, level of education, number of medications, drug interactions and comorbidities in elderly patients.
\end{abstract}

Method: This is a single-center retrospective cohort study conducted in a tertiary hospital in the United Arab Emirates.

Results: A majority of 237 patients aged from 60 to 69 years with average of $67 \pm$ SEM years were included in this study. A total of $89 \%$ of the participated patients were taking more than five medications and exposed to at least one poly-pharmacy episode according to its definition. It was revealed in this study that a greater exposure to poly-pharmacy can lead to co-morbidities. The relationship between age-groups and drug interactions was also measured in this study. The number of drug interactions increased with increasing the number of medications taking by elderly patients. Moreover, the male subjects were more frequently exposed to poly-pharmacy and were found to have a greater number of co-morbidities than their counterpart female subjects. Nevertheless, the highest interventions from healthcare professionals were on patients taking multiple medications and aged 60 to 69 and 70 to 79 years.

Conclusions: This study is additional evidence that the prevalence of poly-pharmacy is widespread among elderly patients. Evaluating the relationship between poly-pharmacy and other factors such as age, gender, level of education, number of medications, drug-drug interactions, interventions and co-morbidities revealed that there is a clear relationship. Therefore, educational programs targeting primary care physicians, other healthcare professionals and elderly patients should be developed in hospital settings.

Keywords: Poly-pharmacy; Elderly patients; Poly-pharmacy in the elderly

\section{Introduction}

Maintaining the health and quality life of elderly patients, is one of the major challenges faced by a society. It is well known that the maximum life expectancy has not changed substantially, but average life expectancy has increased greatly in the past century. This reflects profound improvements in mortality in infancy and young adulthood, resulting in a much greater proportion of people surviving to older ages. Aging group usually places some challenges on healthcare providers to maintain their quality of life. As a result optimizing drug therapy for this specific group of patients becomes an essential part of healthcare. Providing elderly with medications usually goes through a complex process. This include deciding the appropriate drug as per the indication, determining the dose, dosage form, ensuring that the dosing schedule is appropriate for the patients' physiologic status, counseling the patients and monitoring the effectiveness and the side effects of the drugs. Prescribing for elderly patients presents a unique challenge; therefore, poly-pharmacy is an important issue to be addressed [1,2].

According to the published work poly-pharmacy can be simply defined as the use of multiple medications by a patient. According to the published studies, the precise minimum number of medications used to define "poly-pharmacy" is variable, but generally ranges from 5 to 10 [3]. It also refers to the effects of taking multiple medications, mostly more than five medications, to manage co-existing health problems [3]. It is clearly highlighted in that the use of greater number of therapies has been independently associated with increased risk for adverse drug events [4]. This indicates that medications should be provided to elderly patients with caution because of age-related changes in pharmacokinetics and also the preclinical drug trials often include young healthy patients which may not be appropriate for the elderly patients $[5,6]$.

Elderly patients are at greater risk for adverse drug events due to metabolic changes which will be proportionately increased with the number of drugs used. In addition, Poly- 
pharmacy increases the potential for drug-drug interactions and the possibility of "prescribing cascades". Prescribing cascades usually develop when an adverse drug event is misinterpreted as a new medical condition and additional drug therapy is then prescribed to treat this medical condition [7]. In general, the use of multiple medications can lead to many problems such as medication non-adherence, compounded by cognitive or visual issues in elderly patients. Therefore, a balance is required between over-and under-prescribing.

On the other hand, the use of herbal and dietary supplements (such as ginseng, ginkgo, glucosamine and biloba extract) was increased noticeably among elderly patients according to one survey. In this survey 3000 ambulatory adults 75 years of age or older in four states were included. It revealed that almost three quarters of the participated elderly patients used at least one prescription drug and one dietary supplement [8]. According to the published studies, it is clear that herbal medicines may interact with prescribed drug therapies and lead to adverse events, underscoring the importance of routine questioning and educating patients about the use of unconventional therapies. Overall, while poly-pharmacy most commonly refers to prescribed medications, it is important to consider the number of over the counter and herbal/supplements used.

\section{Objectives}

The objective of this study was to assess the relationships between poly-pharmacy and other factors such as age, gender, level of education, number of medications, drug-drug interactions, interventions and co-morbidities in a tertiary hospital in the United Arab Emirates (UAE).

\section{Methods}

This was a single-centre retrospective cohort study conducted in a tertiary hospital in the United Arab Emirates (UAE); the name of the hospital was kept anonymous to comply with the hospital's rules and regulations. This survey was approved by the hospital scientific committee in $22 / 1 / 2014$. This was based on reviewing medical records of patients that complied with the inclusion criteria. The inclusion criteria of this study required all patients to be aged 50 years and above, taking five or more medications and had at least one visit to the hospital in a year. According to the protocol of this study, taking five or more medications by patient aged 50 years or above was considered as poly-pharmacy. The aim and the protocol of this study were explained and discussed with the medical professionals involved in the study in the hospital.

The medical records of the included patients were reviewed and relevant data was collected for 6 months from January to June 2014. The data was analysed using Microsoft Office Excel 2010 and Minitab 17 Statistical Software (made by "Minitab Inc", Pennsylvania, USA). The drug-drug interactions and the severity of these interactions were obtained using Lexicomp Program (made by "Lexi-comp, Inc", Ohio, Hudson). Results of all questions were reported in the data sheet and expressed in \% [95\% Confidence Intervals (CI)].
A total of 237 patients ( $56 \%$ males and $44 \%$ females) complied with the inclusion criteria and were included in this study. Each patient was given a code number and was kept in a separate sheet with the main investigator to retain patients' names anonymous. All elderly patients' records were reviewed and compared to the study inclusion criteria. All medical records that complied with the inclusion criteria were given a code, reviewed and analyzed using a data-entry sheet by a health professional. The data-entry sheet contained twelve questions about the patient code number, age, gender, marital status, nationality, number of medications (including herbal and dietary supplements), name of medications, exact number of chronic medications, drug-drug interactions, co-morbidities, level of education and the interventions from healthcare professionals.

\section{Results}

According to the survey, a total of 133 [(56\% (95\% CI 5063)] males and 104 [44\% (95\% CI, 37-50)] female patients with average age of $67 \pm$ SEM years [range (50-101), median (66)] were included in this study (Table 1). Most of the included older adult patients [40\% (95\% CI, 33-46)] in this study were aged from 60 to 69 years, [18\% (95\% CI, 13-23) were male and $22 \%$ (95\% CI, 16-27) were female]. A total of $42 / 237$ participants [18\% (95\% CI, 13-23)] were highly educated (graduated from college, university or postgraduate), and 195/237 participants [82\% (95\% CI, 77-87)] were less educated (not-educated, graduated from secondary school, vocational training and/or diploma) (Table 1).

It was found in this study that $89 \%,(95 \% \mathrm{CI}, 84-93)$ of the included patients were taking more than five medications and were exposed to at least one poly-pharmacy episode. About quarter $[25 \%,(95 \% \mathrm{CI}, 19-31)]$ of them were taking between 1 and 6 medications, [11\%, (95\% CI, 07-16) taking between 1-4 medications and 14\% (95\% CI, 10-19) were taking between 5 and 6 medications]. Out of the total included patients, 24\% (95\% CI, 19-30) were taking around 7 to 9 medications and more than half [51\%, $(95 \% \mathrm{CI}, 45-58)]$ were taking more than 9 medications. Calculating the average of co-morbidities in this study showed that co-morbidities substantially increase with age. The highest co-morbidity was observed in patients aged from 6069 years, as shown in Figure 1. This study has also revealed that the prevalence of poly-pharmacy substantially increases with age. A higher exposure to poly-pharmacy was reflected in the age group 60 to 79 years [ $56 \%$ ( $95 \% \mathrm{CI}, 50-63)$ ], as compared to the other age groups. For the age group of 60 to 69 years and 70 to 79 years it was $34 \%$ (95\% CI, 28-40) and 23\% (95\% CI, 17-28) respectively.

In addition, the 60 to 79 years age group also had the maximum number of incidences of consuming more than 9 medications [35\% (95\% CI, 28-41)]. The age group 60 to 69 years reported an incidence of $19 \%$ (95\% CI, 14-24) and the age group 70 to 79 years reported that of $16 \%$ ( $95 \%$ CI, 12-22), as depicted in Figure 2.

When comparing poly-pharmacy with non-poly-pharmacy among the participated age groups, 60 to 69 and 70 to 79 age 
Table 1: Demographics of the participating patients ( $\mathrm{n}=237)$.

\begin{tabular}{|c|c|c|c|}
\hline Demographics & $\begin{array}{l}\text { Number of } \\
\text { Responders }\end{array}$ & $\begin{array}{c}\text { Percentages } \\
\text { (\%) of } \\
\text { Responders }\end{array}$ & $\begin{array}{c}95 \% \\
\text { Confidence } \\
\text { Intervals }\end{array}$ \\
\hline \multicolumn{4}{|l|}{ Gender $\left(n^{*}=237\right)$} \\
\hline Male & 133 & 56 & $(50-63)$ \\
\hline Female & 104 & 44 & $(37-50)$ \\
\hline \multicolumn{4}{|c|}{ Age distribution $\left(\mathrm{n}^{*}=237\right)$} \\
\hline $50-59$ years & 59 & 25 & $(20-31)$ \\
\hline $60-69$ years & 93 & 39 & $(33-46)$ \\
\hline 70-79 years & 57 & 24 & $(19-30)$ \\
\hline 80-89 years & 20 & 8 & $(05-13)$ \\
\hline$\geq 90$ years & 08 & 03 & $(01-07)$ \\
\hline \multicolumn{4}{|c|}{ Number of medications taken daily $\left(n^{*}=234\right)$} \\
\hline 1-4 medications & 26 & 11 & $(07-16)$ \\
\hline 5-6 medications & 32 & 14 & $(10-19)$ \\
\hline 7-9 medications & 56 & 24 & $(19-30)$ \\
\hline$>9$ medications & 120 & 51 & $(45-58)$ \\
\hline \multicolumn{4}{|c|}{ The level of education $\left(n^{*}=237\right)$} \\
\hline Not Educated & 58 & 24 & $(19-30)$ \\
\hline Secondary School & 87 & 37 & $(31-43)$ \\
\hline Diploma & 50 & 21 & $(16-27)$ \\
\hline College & 34 & 14 & $(10-19)$ \\
\hline Postgraduate & 08 & 04 & $(0.14-07)$ \\
\hline
\end{tabular}

groups showed the highest poly-pharmacy exposures [30\% (95\% CI, 25-37) and 22\% (95\% CI, 17-28)], respectively. However, age groups that showed lowest levels of poly-pharmacy were 80 to 89 years [8\% (95\% CI, 05-12)] and greater than 90 years $[2.53 \%$ (95\% CI, 01-05)], respectively. In addition, poly-pharmacy was prevalent in $46 \%(95 \% \mathrm{CI}, 40-53)$ of the elderly males as compared to $37 \%$ females (95\% CI, 31-43). Moreover, when comparing the number of medications' intake with respect to genders, it was found that males had more number of medications than their counterpart females in all age groups.

When measuring the relationships between age groups and drug interactions, it was revealed that the number of drug interactions increased with increasing the number of medications intakes. For example, when 38.92\% (95\% CI, 37- 41) of medications were taken by 60-69 years age group, the drug interactions reached its highest at $38.41 \%$ (95\% CI, 36-41) and when 3.34\% (95\% CI, 03-04) of medications were taken by the age group 'greater than 90 years', the drug interactions reached its lowest at $2 \%$ (95\% CI, 1.4-03). Most of the drug interactions were reported in 60 to 79 years age group [38.92\% (95\% CI, $37-41$ )] and the 70 to 79 years age group [28.34\% (95\% CI 26$30)]$. Moreover, more than half [56\% (95\% CI 51-59)] of patients that were most commonly involved in poly-pharmacy, had comorbidities of high blood pressure, diabetes and dyslipidemia [23\% (95\% CI, 20-26), 18\% (95\% CI, 16-21) and 15\% (95\% CI, 13-18) respectively. It was also shown that co-morbidities increases with the increase of the number of medications taking by patients. On the other hand, there was no clear relationship between the level of education and co-morbidities shown in this study.

This study had clearly shown a clear relationship between poly-pharmacy and co-morbidities, as shown in Figure 3. The more medications a patient consumed, the greater was the exposure to co-morbidity. This has led to an increased intervention by the healthcare professionals, such as physicians and pharmacists. For example, it was found in this study that the healthcare providers' interventions increased in parallel with the increased number of medications as well as with poly-pharmacy. Precisely, the highest healthcare provider intervention was for patients taking more than 9 medications [62\% (95\% CI, 54-69)]. In addition, the obvious interventions were for patients aged 60 to 69 [38\% (95\% CI, 31-45] and 70 to 79 years [27\% (95\% $\mathrm{CI}, 21-34)]$. Interventions included substituting medications because of their side-effects, changing doses and dosage forms to ensure compliance and adherence and sometimes removing medications to decrease drug-drug interactions. Nevertheless, it was revealed that there were no direct relationships between the level of the healthcare interventions and co-morbidities.

Moreover, it was also revealed that the intervention by healthcare professionals increases with the less educated patients. For example, 65\% (95\% CI, 58-72) was the highest intervention and was for the uneducated and patients with secondary school education [38\% (95\% CI, 31-46) and $27 \%$ (95\% CI, 21-34) respectively]. In addition, it was shown that $66 \%$ (95\% CI, 63-70) of the less educated patients had the most comorbidities [30\% (95\% CI, 27-33)] for the uneducated patients and $[36 \%,(95 \%$ CI, 33-40)] for the secondary educated patients. This study had also shown that the number of medications taken by patients decreased with the higher level of education. Elderly patients who were graduated from colleges were taking an average of 8 medications compared to not-educated patients who were taking an average of 11 medications.

\section{Discussion}

Evaluating the relationship between poly-pharmacy and other factors such as age, gender, level of education, number of medications, drug-drug interactions, interventions and comorbidities revealed that there is a clear relationship between poly-pharmacy and most of these factors. For example, it was revealed in this study that there was a clear relationship between poly-pharmacy and age; the higher the number of medications found, the greater the risk of poly-pharmacy. It was indicated that $89 \%$ (95\% CI, 84-93) of elderly patients, mostly aged from 60 to 79 years old, were taking more than five medications and were exposed to at least one poly-pharmacy episode. In addition, the 60 to 79 years age group also had the maximum number of incidences of consuming more than 9 medications [35\% (95\% CI, 28-41)]. This shows that the issue of poly-pharmacy can be of particular concern in older people as compared to the younger individuals, individuals which can be related to the disease conditions for which therapies were prescribed [9].

Poly-pharmacy was also clearly shown in elderly patients 


\section{Age Group and Comorbidities}

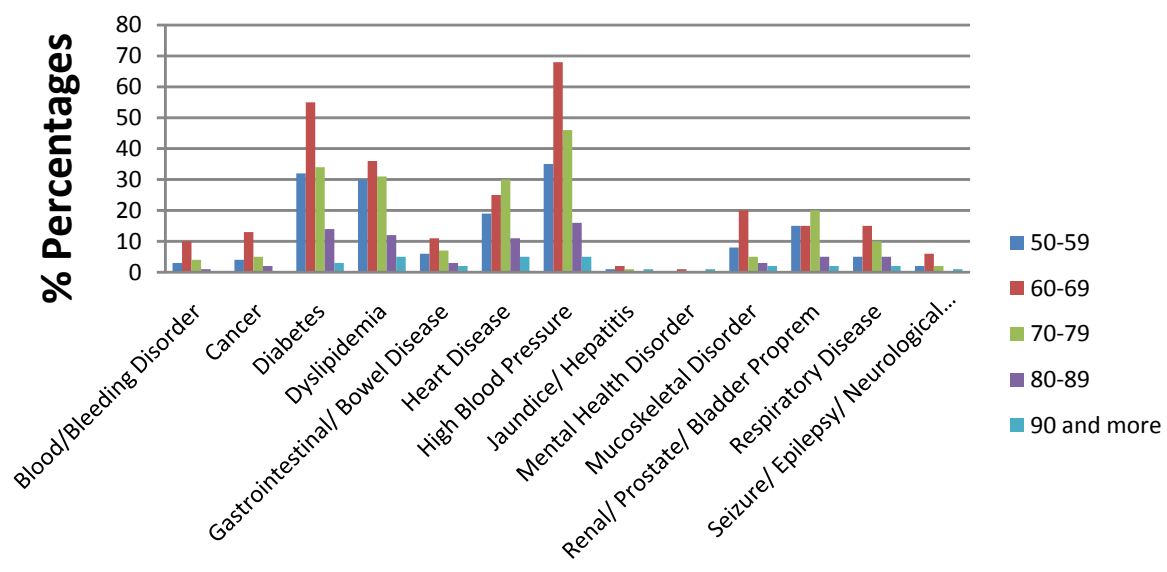

\section{Comorbidities}

Figure 1: The distribution of co-morbidities among age groups.

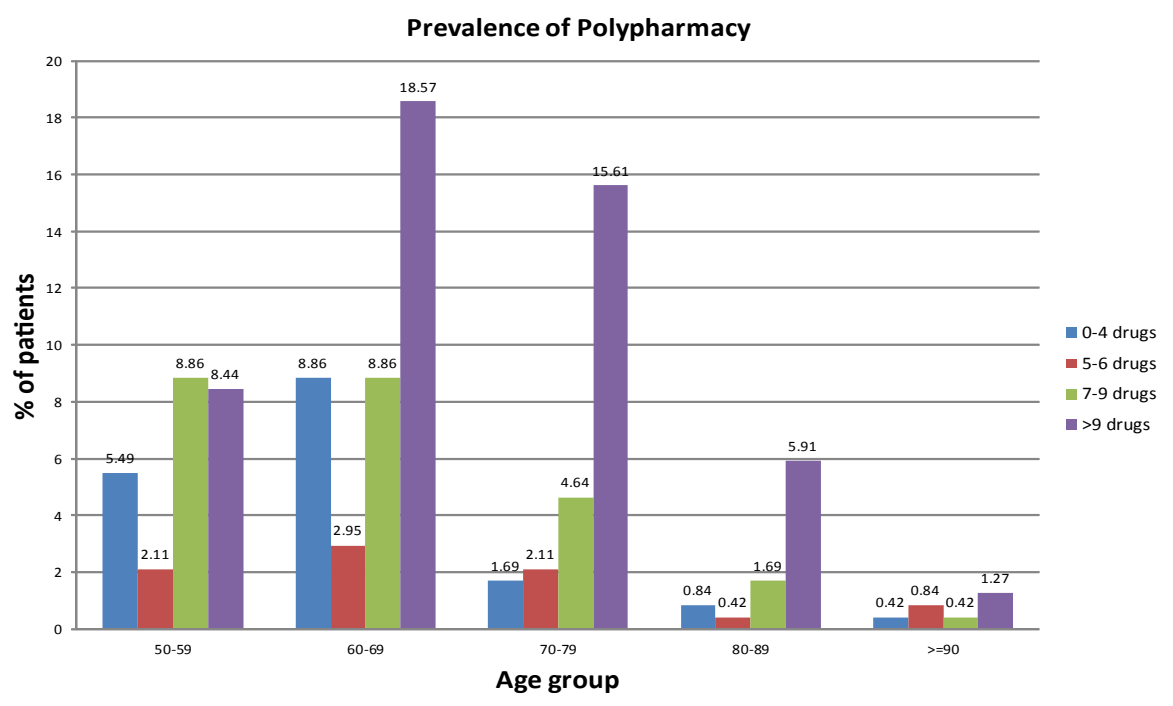

Figure 2: Prevalence of poly-pharmacy among the participating age groups.

aged 60 to 69 and 70 to 79 years and the lowest poly-pharmacy were shown in age groups 80 to 89 and 'greater than 90 years' old. This result can be related to the fact that the older ages 80 to 'greater than 90 years' old were healthy patients and were taking less medications and showed less co-morbidities. In addition, it was shown in this study that elderly males were exposed to polypharmacy more than elderly females from the same age groups. This can also be related to the fact that elderly males were taking more medications than elderly females in all age groups and showed more co-morbidities (such as diabetes, cardiovascular disease, stroke and cancer). However, some study from the literature had shown that there is no association between polypharmacy and gender or age [10].
It was also revealed in this study that co-morbidities were highest in older age groups from 60-79 which showed highest exposure to poly-pharmacy. In addition, it was shown that the highest co-morbidities correlated with poly-pharmacy were high blood pressure, diabetes and dyslipidemia. A total percentage of $56 \%$ (95\% CI, 51-59) of patients were involved in poly-pharmacy and showed co-morbidities of high blood pressure, diabetes and dyslipidemia [23\% (95\% CI, 20-26), 18\% (95\% CI, 16-21) and 15\% (95\% CI, 13-18) respectively].

Furthermore, there was a clear relationship found between poly-pharmacy and co-morbidities. According to the literature, there are many reasons associated with elderly to be exposed for poly-pharmacy. Those included that elderly are at greater 


\section{Polypharmcy and Comorbidities}

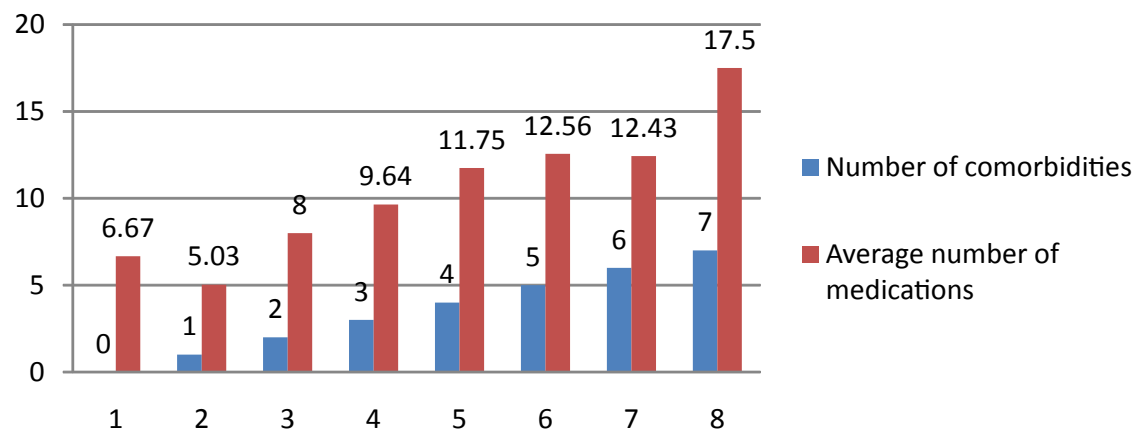

Figure 3: The relationship between poly-pharmacy and patient's co-morbidities.

risk for adverse drug events due to metabolic changes and decreased drug clearance associated with aging which are compounded by increasing numbers of drugs used. In addition, poly-pharmacy increases the potential for drug-drug interactions and for prescribing inappropriate medications [11]. For example, when measuring the relationships between age group and drug interactions in this study; it was found that the number of drug interactions increased with the increased number of medications. It was shown that when $38.92 \%$ (95\% CI, 37-41) of medications were taking by 60 to 69 years age group, the drug interactions reached its highest at $38.41 \%$ (95\% CI, 36-41). This can be related to that the more intakes of medications by elderly patients can lead to more drug-drug interactions. For example, a study from the literature showed that there is a clear association between the increased use of medications (poly-pharmacy) and unplanned rehospitalisation [12]. Another study has clearly established a strong relationship between poly-pharmacy and negative clinical outcomes [1]. Overall, many studies in the literature highlighted that adverse medical effects occur more frequently with elderly patients due to factors, such as age-specific metabolic changes, issues with compliance, and adherence. Therefore, a welldesigned inter-professional supervision and close monitoring is essential, for this group of patients to reduce aspects of unnecessary prescribing, drug-drug interactions and negative results on health outcomes $[9,13]$.

The level of education, knowledge, severity of the disease and the involvement of patients in decisions regarding their health plays a major role in framing patients' risks of poly-pharmacy. For example, a total of $18 \%$ (95\% CI, 13-23) of patients in the present study were considered highly educated (graduated from college, university and/or postgraduate), and $82 \%$ (95\% CI, 77-87) were considered less educated (non-educated, graduated from secondary school, vocational training or diploma). According to this study, patients with higher level of education were found more knowledgeable about their medications and health status; as a result they were more likely to be involved in self-monitoring and accepting healthcare interventions to avoid poly-pharmacy. On the other hand, the elderly with less education were less knowledgeable about their medications and health status; therefore, they were more susceptible to poly-pharmacy.
It was highlighted in this study that the number of medications taken by elderly decreases with increased level of education. For example, elderly with high level of education were taking an average of 8 medications compared to non-or-less educated patients who were taking an average of 11 medications. In addition, it was found that the highest interventions by healthcare professionals were with the less educated patients compared to the more educated ones. However, it was revealed that there were no direct relationships between the level of the healthcare interventions and the co-morbidities and between the level of education and co-morbidities. On the other hand, the healthcare interventions increased with increasing the number of medications (poly-pharmacy) and comorbidities. For example, it was shown that the highest intervention [62\% (95\% CI, 5469)] was for patients taking more than 9 medications.

Elderly patients are particularly vulnerable to drug-drug interactions because they often have multiple chronic medical conditions requiring multiple drug therapies. The risk of an adverse event due to drug-drug interactions is substantially increased when multiple drugs are taken $[14,15]$. For example, the risk of bleeding for elderly on warfarin is increased with co-administration of selective and non-selective NSAIDs, SSRIs, omeprazole, lipid-lowering agents, amiodarone, and fluorouracil [14]. Periodic evaluation of a patient's drug regimen is an essential component of medical cares for all patients and in particular the elderly. Such a review may indicate the need for changes in prescribing a drug therapy. These interventions may include discontinuing a therapy prescribed for an indication that no longer exists, substituting a therapy with a therapeutically equivalent counterpart, changing drug doses and/or dosage forms and appropriately adding a new medication.

Clinicians in all practices should consider medication review and dosing adjustment for elderly patients to detect any changes in patient status such as renal or liver impairments. In addition, considering adverse drug events for any new symptom before adding a new therapy to the patient's drug regimen is very essential to detect any presentation of an atypical adverse drug effect to an existing drug therapy [16]. Another reasonable approach to apply proper and effective medication review, newly diagnosed patients should be scheduled to visit their physician 
within a limited time after starting a new medication. Putting in mind that patients may not consider over-the-counter products, ointments, vitamins, ophthalmic preparations, or herbal medicines as drug therapies and hence they should be asked to bring all such products to the clinician during their hospital visits. A qualitative research conducted on 18 elderly men aged over 60 years found that there is high consumption of herbals among the elderly especially in the treatment of minor harm assuming that they are natural and show few side effects. They were found using herbals concurrently with the manufactured medicines without regard to risk of interactions and without reporting to health professionals [17]

Nevertheless, when drug therapy is indicated for the elderly patient, it may be possible to substitute a safer alternative for the current regime. Many adverse drug events are dose-related; when prescribing drug therapies it is important to use the minimal dose required to obtain clinical benefit [18]. A survey conducted in the UAE to evaluate the level of interaction between physicians and community pharmacists revealed that $60 \%$ of physicians rarely or never discussed patients' drug therapy with pharmacists [19] Overall, when multiple medications are required, greater regimen complexity will increase the likelihood of poor compliance or confusion with dosing. Elderly patients, and particularly those with low health literacy, are not able to efficiently consolidate prescription regimens to optimize a dosing schedule.

Particular care must be taken in determining drug dosages when prescribing for elderly patients. Higher dosages and decreased clearance usually prolong drug's half-life which will lead to increased plasma drug concentrations in older people. Multiple factors contribute to the appropriateness and general quality of drugs that are being prescribed. Those include avoidance of inappropriate medications, appropriate use of medications, monitoring for side effects and drug levels, avoidance of drug-drug interactions, and involvement of the patient and integration of the patient values. Approaches to decrease inappropriate prescribing in elderly patients include educational interventions, computerized order entry and decision support and multidisciplinary team care led by physicians and/ or pharmacists [2].

Nevertheless, the extent of medical and financial problems of poly-pharmacy is alarming particularly in elderly patients. Many studies conducted to evaluate the effect of professionals' interventions to improve drug therapy and minimize drug intake in elderly patients declared that the intervention was associated with a substantial decrease in the cost of drugs [20$25]$. For example, a survey in the UAE revealed that $95 \% \mathrm{CI}$ 51-66) of participated patients believed that they should be consulted and monitored when switching their medicines for better clinical outcomes and for better cost management [25]. Another survey evaluating the level of interaction between physicians and community pharmacists in the UAE revealed that $60 \%$ of physicians rarely or never discussed patients' drug therapy with pharmacists [19]. This explores the clear need for monitoring and educating elderly patients, particularly in terms of clarifying the prevalence of poly-pharmacy and the potential roles of healthcare professionals in successfully introducing new and reviewing existing drug therapy.

\section{Conclusions}

This study is additional evidence that the prevalence of polypharmacy is widespread among elderly patients. Evaluating the relationship between poly-pharmacy and other factors such as age, gender, level of education, number of medications, drugdrug interactions, interventions and co-morbidities revealed that there is a clear relationship. Therefore, a step-wise approach should be developed in all hospitals to decrease the exposure of elderly patients to poly-pharmacy. Thus, inclusive educational programs targeting primary care physicians, other healthcare professionals and elderly patients should be developed. This will ensure safe, effective and appropriate use of medications in these specific populations. Strategies should also be defined to closely monitor elderly who are more likely to be exposed to polypharmacy to increase the awareness of the magnitude of polypharmacy phenomenon, improve drug therapy and minimize drug intake in elderly patients with a substantial decrease in the cost of drugs.

\section{Limitations}

Limitations of this study include that the attitudes toward poly-pharmacy was mainly assessed from reviewing patients' medical records and not from interviewing patients or healthcare professionals.

\section{Acknowledgement}

Authors are grateful to all healthcare professionals for supporting and welcoming the results of this study. We are also thankful to the government of the United Arab Emirates for supporting and encouraging independent scientific research.

\section{Competing interest}

This study was self-funded by the authors and they have no financial or proprietary interest in the subject matter or material discussed.

\section{References}

1. Maher RL, Hanlon J, Hajjar ER. Clinical consequences of polypharmacy in elderly. Expert Opin Drug Saf, 2014; 13(1): 57-65. doi: 10.1517/14740338.2013.827660.

2. Kim HA, Shin JY, Kim MH, Park BJ. Prevalence and predictors of polypharmacy among Korean elderly. PLoS One. 2014; 9(6): e98043. doi: 10.1371/journal.pone.0098043.

3. Ferner RE, Aronson JK. Communicating information about drug safety. BMJ. 2006; 333(7559): 143-145. doi: 10.1136/bmj.333.7559.143.

4. Field TS, Gurwitz JH, Avorn J, McCormick D, Jain S, Eckler M, et al. Risk factors for adverse drug events among nursing home residents. Arch Intern Med. 2001; 161(13): 1629-34.

5. Cho S, Lau SW, Tandon V, Kumi K, Pfuma E, Abernethy DR. Geriatric drug evaluation: Where are we now and where should we be in the future? Arch Intern Med. 2011; 171(10): 937-940. doi: 10.1001/ archinternmed.2011.152.

6. Mojtabai R, Olfson M. National trends in psychotropic medication 
polypharmacy in office-based psychiatry. Arch Gen Psychiatry. 2010 67(1): 26-36. doi: 10.1001/archgenpsychiatry.2009.175.

7. Rochon PA, Gurwitz JH. Optimising drug treatment for elderly people: the prescribing cascade. BMJ. 1997; 315(7115): 1096-1099.

8. Nahin RL, Pecha M, Welmerink DB, Sink K, DeKosky ST, Fitzpatrick AL et al. Concomitant Use of Prescription Drugs and Dietary Supplements in Ambulatory Elderly People. J Am Geriatr Soc. 2009; 57(7): 11971205. doi: 10.1111/j.1532-5415.2009.02329.x.

9. Eckardt R, Steinhagen-Thiessen E, Kämpfe S, Buchmann N Polypharmazie und Arzneimitteltherapiesicherheit im Alter. Zeitschrift für Gerontologie und Geriatrie. 2014; 47(4): 293-301. doi: 10.1007/s00391-013-0562-0.

10. Lucchetti G, Granero AL, Pires SL, Gorzoni ML. Factors associated to polypharmacy in institutionalized elderly. Revista Brasileira de Geriatria e Gerontologia. 2010; 13: 51-58.

11. Weng MC, Tsai CF, Sheu KL, Lee YT, Lee HC, Tzeng SL, et al. The impact of number of drugs prescribed on the risk of potentially inappropriate medication among outpatient older adults with chronic diseases. QJM. 2013; 106(11): 1009-1015. doi: 10.1093/qjmed/hct141.

12. Morandi A, Bellelli G, Vasilevskis EE, Turco R, Guerini F, Torpilliesi T, et al. Predictors of Rehospitalization Among Elderly Patients Admitted to a Rehabilitation Hospital: The Role of Polypharmacy, Functional Status, and Length of Stay. J Am Med Dir Assoc. 2003; 14(10): 761767. doi: 10.1016/j.jamda.2013.03.013.

13. Arruda GO, Lima SC, Renovato RD. The use of medications by elderly men with polypharmacy: representations and practices. Rev Lat Am Enfermagem. 2013; 21(6): 1337-1344. doi: 10.1590/01041169.3004.2372.

14. Holbrook AM, Pereira JA, Labiris R, McDonald H, Douketis JD, Crowther M, et al. Systematic overview of warfarin and its drug and food interactions. Arch Intern Med. 2005; 165(10): 1095-1106.

15. Juurlink DN, Mamdani M, Kopp A, Laupacis A, Redelmeier DA. Drugdrug interactions among elderly patients hospitalized for drug toxicity. JAMA. 2003; 289(13): 1652-1658.
16. Steinman MA, Hanlon JT. Managing medications in clinically complex elders: "There's got to be a happy medium". JAMA. 2010; 304(14): 1592-1601. doi: 10.1001/jama.2010.1482.

17. Lima SC, Arruda GO, Renovato RD, Alvarenga MR. Representations and uses of medicinal plants in elderly men. Rev Lat Am Enfermagem. 2012; 20(4): 778-786.

18. Turner MR, Camacho X, Fischer HD, Austin PC, Anderson GM, Rochon $\mathrm{PA}$, et al. Levothyroxine dose and risk of fractures in older adults: nested case-control study. BMJ. 2011; 342: d2238. doi: 10.1136/bmj. d2238.

19. Abduelkarem AR, Sharif SI. Current levels of interaction between the physician and pharmacist: A comparative study in Libya and UAE. Jordan Journal of Pharmaceutical Sciences. 2008; 1(2): 142- 151.

20. Burns C. IMS Health Study Identifies $\$ 200+$ Billion Annual Opportunity from Using Medicines More Responsibly.

21. Eisenberg DM, Kessler RC, Foster C, Norlock FE, Calkins DR, Delbanco TL. Unconventional Medicine in the United States. Prevalence, Costs, and Patterns of Use. N Engl J Med. 1993; 328(4): 246-252.

22. Al Ameri MN, Whittaker C, Tucker A, Yaqoob M, Johnston A. A survey to determine the views of renal transplant patients on generic substitution in the UK. Transpl Int. 2011; 24(8): 770-779. doi: 10.1111/j.1432-2277.2011.01268.

23. Johnston A, Stafylas P, Stergiou GS. Effectiveness, safety and cost of drug substitution in hypertension. Br J Clin Pharmacol. 2010; 70(3): 320-334. doi: 10.1111/j.1365-2125.2010.03681.x.

24.Johnston A. Challenges of therapeutic substitution of drugs for economic reasons: focus on CVD prevention. Curr Med Res Opin. 2010; 26(4): 871-8. doi: 10.1185/03007990903578462.

25. Al Ameri MN, Mohamed W, Makramalla E, Shalhoub B, Tucker A JA. Renal patients' views on generic prescribing and substitution: Example from the United Arab Emirates. East Mediterr Health J. 2013; 19(4): 373-81. 